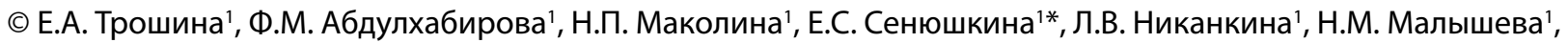
И.Н. Репинская ${ }^{2}$ В.А. Дивинская ${ }^{2}$

'Национальный медицинский исследовательский центр эндокринологии, Москва, Россия

${ }^{2}$ Министерство здравоохранения Республики Крым, Симферополь, Россия

ОБОСНОВАНИЕ. В статье представлены результаты проведенного в сентябре 2020 г. специалистами ФГБУ «НМИЦ эндокринологии» Минздрава России контрольно-эпидемиологического исследования, направленного на оценку современного состояния йодной обеспеченности населения Республики Крым. Исследование в Крыму входит в ряд мероприятий и работ, выполняемых по поручению Министерства здравоохранения РФ в рамках государственных заданий «Научная оценка необходимости принятия дополнительных нормативных правовых и иных мер по ликвидации йодного дефицита в пилотных регионах с тяжелым йодным дефицитом» и «Эпидемиологические и молекулярно-клеточные характеристики опухолевых, аутоиммунных и йододефицитных тиреопатий как основа профилактики осложнений и персонализации лечения».

Полученные данные отображают состояние проблемы потребления йода на территории Крымского полуострова и свидетельствуют об актуальности принятия региональной профилактической программы, направленной на устранение дефицита йода в питании населения и связанных с ним заболеваний.

ЦЕЛЬ. Оценка йодной обеспеченности населения Республики Крым.

МАТЕРИАЛЫ И МЕТОДЫ. Исследование проводилось в общеобразовательных школах четырех районов Республики Крым - в гг. Симферополь, Белогорск, Бахчисарай и Саки.

Объем исследования - 356 школьников 8-10 лет, всем было выполнено: сбор анамнеза и антропометрических показателей (рост, вес), осмотр врача-эндокринолога с пальпацией щитовидной железы (ЩЖ), ультразвуковое исследование (УЗИ) ЩЖ, получение разовых порций мочи и образцов пищевой поваренной соли (5-10 г), которая ежедневно используется в питании в их семьях. Измерения роста и веса детей стандартной методикой проводились во время осмотра специалистом. УзИ щЖ выполнялось в положении лежа с использованием портативного ультразвукового аппарата LOGIQе (China) с мультичастотным линейным датчиком 10-15 МГц. Все образцы мочи

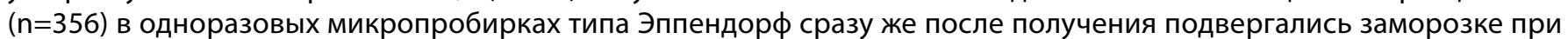
температуре $-20-25^{\circ} \mathrm{C}$ для дальнейшего определения концентрации йода в моче с помощью церий-арсенитного метода (на базе клинико-диагностической лаборатории ФГБУ «НМИЦ эндокринологии» Минздрава России). Качественное исследование на наличие йодата калия в образцах пищевой поваренной соли $(\mathrm{n}=203)$ осуществлялось на месте экспресс-методом.

От всех родителей/опекунов детей получены информированные согласия на проведение обследования и обработку персональных данных. Разрешение локального этического комитета ФГБУ «НМИЦ эндокринологии» Минздрава России получено 25 марта 2020 г., №5.

РЕЗУЛЬТАТЫ. По результатам обследования 356 детей младшего школьного возраста медианная концентрация йода в моче (мКЙМ) составляет 97 мкг/л и варьирует от 78 до 98 мкг/л в обследованных районах, доля проб мочи со сниженной концентрацией йода составила 51,2\%. Доля использования в семьях школьников йодированной соли составила 12,3\% (диапазон значений от 10 до 15\%). Средняя частота распространенности зоба у детей по данным УзИ 9,5\% (диапазон значений от 1,7 до 16,3\%).

\title{
ЗАКЛЮЧЕНИЕ.
}

1. Показатель медианной концентрации йода в моче свидетельствует о недостаточной йодной обеспеченности населения Республики Крым.

2. Распространенность зоба у детей младшего школьного возраста по данным УЗИ ЩЖ соответствует легкой степени тяжести йодного дефицита в регионе.

3. Доля домохозяйств, использующих йодированную соль, крайне низкая, и составляет 12,3\%, что не соответствует рекомендациям ВОЗ для регионов с природным дефицитом йода (от $90 \%$ и более).

КЛЮЧЕВЫЕ СЛОВА: йодный дефицит; зоб; йододефицитные заболевания; йодированная соль. 


\section{IODINE DEFICIENCY DISORDERS: CURRENT STATE OF THE PROBLEM IN THE REPUBLIC OF CRIMEA}

Ekaterina A. Troshina', Fatima M. Abdulkhabirova', Natalya P. Makolina', Evgeniya S. Senyushkina ${ }^{1 *}$, Natalia M. Malysheva', Irina N. Repinskaya² ${ }^{2}$ Valentyna A. Divinskaya²

'Endocrinology Research Centre, Moscow, Russia

${ }^{2}$ Ministry of Health of the Republic of Crimea

BACKGROUND: The article presents the results of a control and epidemiological study conducted in September 2020 by specialists of the Federal State Budgetary Institution «NMIC of Endocrinology» of the Ministry of Health of Russia, aimed at assessing the current state of iodine provision in the population of the Republic of Crimea. The study in Crimea is part of a number of activities and work carried out on behalf of the Ministry of Health of the Russian Federation within the framework of state assignments «Scientific assessment of the need for additional regulatory legal and other measures to eliminate iodine deficiency in pilot regions with severe iodine deficiency» and «Epidemiological and molecular-cellular characteristics of tumor, autoimmune and iodine deficiency thyropathies as a basis for prevention of complications and personalization of treatment.

The data obtained reflect the state of the problem of iodine consumption on the territory of the Crimean Peninsula and indicate the relevance of the adoption of a regional preventive program aimed at eliminating iodine deficiency in the diet of the population and related diseases.

AIM: Assessment of iodine supply of the population of the Republic of Crimea

MATERIALS AND METHODS: The research was carried out in secondary schools of four districts of the Republic of Crimea in the years. Simferopol, Belogorsk, Bakhchisarai and Saki.

The volume of the study - 356 schoolchildren of 8-10 years old, all were completed: taking anamnesis and anthropometric parameters (height, weight), examination by an endocrinologist with palpation of the thyroid gland (thyroid gland), ultrasound examination of the thyroid gland (thyroid ultrasound), obtaining single portions of urine and samples of table salt (5-10 grams), which is used daily in the diet in their families. The measurements of the height and weight of the children by the standard method were carried out during the examination by a specialist. Thyroid ultrasound was performed in the supine position using a portable LOGIQe ultrasound machine (China) with a 10-15 MHz multifrequency linear transducer. All urine samples $(n=356)$ in disposable Eppendorf microtubes were immediately frozen at a temperature of minus $20-25^{\circ}$ ! for further determination of the concentration of iodine in urine using the cerium-arsenite method (based on the clinical diagnostic laboratory of the Federal State Budgetary Institution NMITs endocrinology «of the Ministry of Health of Russia). A qualitative study for the presence of potassium iodate in food table salt samples $(n=203)$ was carried out on site by the express method.

Informed consent was obtained from all parents / guardians of children for the examination and processing of personal data. The permission of the local ethical committee of the Federal State Budgetary Institution «NMIC of Endocrinology» of the Ministry of Health of Russia was received on March 25, 2020, No. 5.

RESULTS: According to the results of a survey of 356 children of primary school age, the median concentration of iodine in urine (mCIM) is $97 \mu \mathrm{g} / \mathrm{I}$ and varies from 78 to $98 \mu \mathrm{g} / \mathrm{I}$ in the surveyed areas, the proportion of urine samples with a reduced iodine concentration was $51.2 \%$. The share of iodized salt use in the families of schoolchildren was $12.3 \%$ (values range from $10 \%$ to $15 \%)$. The average incidence of goiter in children according to ultrasound data is $9.5 \%$ (range of values from $1.7 \%$ to $16.3 \%$ ).

\section{CONCLUSION.}

1. The indicator of the median concentration of iodine in urine indicates an insufficient iodine supply of the population of the Republic of Crimea.

2. The prevalence of goiter in children of primary school age according to the ultrasound examination of the thyroid gland corresponds to the mild severity of iodine deficiency in the mild degree of goiter endemic in the region.

3. The share of households using iodized salt is extremely low and amounts to $12.3 \%$, which does not meet the WHO recommendations for regions with natural iodine deficiency (from $90 \%$ or more).

KEYWORDS: iodine deficiency; goiter; iodine deficiency diseases; iodized salt.

\section{ОБОСНОВАНИЕ}

Йододефицитные заболевания (ЙДЗ) представляют собой серьезную проблему общественного здравоохранения во всем мире. Йодный дефицит является единственной и наиболее распространенной причиной поражения головного мозга и нарушения психического развития (www.who.int/ru). Стоит отметить, что давно известны пути решения данной проблемы, и последствия, связанные с дефицитом йода, можно предупредить. Мировой опыт показал, что ключевая составляющая в достижении успеха формируется только при содействии в решении проблемы ЙДЗ органов государственной власти и закреплении принятых решений на законодательном уровне [1-3].

Согласно постановлению Правительства Российской Федерации от 05.10.1999 г. №1119 «О мерах по профилактике заболеваний, вызванных дефицитом йода», были разработаны региональные программы профилактики ЙД3, но далеко не во всех субъектах страны они обновлены с 2000 г. и действуют по настоящее время. По данным 
Глобальной сети по йоду (lodine Global Network), Российская Федерация относится к районам с умеренным дефицитом йода, медианная концентрация йода в моче (мКЙМ) составляет 78 мкг/л, поэтому проблема йодной обеспеченности населения России остается нерешенной на большей части территории нашей страны [4]. В 2019 г. были внесены изменения в СанПиН 2.4.5.2409-08 «Санитарно-эпидемиологические требования к организации питания обучающихся в общеобразовательных учреждениях, учреждениях начального и среднего профессионального образования» в части использования при приготовлении блюд и кулинарных изделий соли поваренной пищевой йодированной. Однако этих мер недостаточно, важно, что принятые и реализованные во многих странах законы предусматривают йодирование наиболее широко потребляемых в пищу сортов соли, обязательное использование йодированной соли в пищевой промышленности, прежде всего в хлебопечении. Данные мероприятия ведут к существенному улучшению здоровья населения и устойчивому обеспечению адекватного йодного потребления [1-3].

Оценка выраженности йодного дефицита при проведении эпидемиологических исследований осуществляется по трем основным параметрам $[1,5]$.

1. Экскреция йода с мочой - основной эпидемиологический показатель, характеризующий обеспеченность йодом населения; является главным критерием оценки тяжести йодного дефицита. Примерно 90\% йода выводится с мочой, поэтому концентрация йода в моче достаточно точно отражает величину потребления йода с пищей. Данный показатель имеет важнейшее значение не только для характеристики эпидемиологической ситуации, но и для осуществления контроля программ профилактики ЙДЗ.

Экскреция йода с мочой является количественным и прямым показателем йодной обеспеченности населения в целом. Содержание йода в моче непригодно для оценки индивидуального потребления йода ввиду высокой межиндивидуальной вариабельности. В результате проводимых эпидемиологических исследований определяется мКЙМ (средняя величина в ряду), что позволяет пренебречь очень неравномерным распределением уровня йода в образцах мочи.

2. Распространенность зоба - клинический количественный непрямой показатель выраженности йодного дефицита. Оценка степени распространенности зоба отражает прежнюю (но не существующую в данный момент) обеспеченность населения йодом, так как в условиях йододефицита для развития зоба требуется не менее 2-3 лет. Учитывая низкую чувствительность и специфичность пальпации щитовидной железы (ЩЖ) для определения степени зоба, в рамках эпидемиологических исследований рекомендуется проведение ультразвукового исследования (УЗИ) ЩЖ.

3. Надежным критерием эффективности профилактических программ является определение доли населения, потребляющего йодированную соль. Эти сведения часто получают путем опроса населения, но наиболее информативным подходом является качественное (или количественное) определение йода в образцах соли, собранных в домохозяйствах. При превышении данного показателя более $90 \%$ профилактические мероприятия признаются эффективными.
Для проведения эпидемиологического исследования важную роль играет выбор репрезентативной группы. Наиболее неблагоприятное воздействие йодный дефицит оказывает на детей и женщин детородного возраста. Наиболее целесообразно как с научной, так и организационной точки зрения проводить эпидемиологическое обследование детей младшего школьного возраста (8-10 лет), так как в этот период наличие зоба связано преимущественно с дефицитом йода. Основой выбора репрезентативной группы является кластерное исследование, проводимое на базе школ.

Распространенность заболеваний ЩЖ продолжает неуклонно увеличиваться, это характерно для Российской Федерации в целом и для Республики Крым в частности. Согласно данным Росстата, общая заболеваемость патологией щЖ в Республике Крым составляет 3228,9 случая на 100 тыс. детского населения и 3167,2 случая на 100 тыс. взрослого населения за 2017-2019 гг., на ЙДЗ приходится 2622,8 и 1049,9 случая соответственно; таким образом, в структуре первичной и общей заболеваемости болезнями ЩЖ среди детского населения первое место занимает эндемический зоб, связанный с йодной недостаточностью, второе место - иные формы нетоксического зоба [6-8].

Уровень тиреотропного гормона (ТТГ) у новорожденных может быть чувствительным маркером йодного дефицита при использовании порогового уровня ТТГ > 5 мкЕД/мл. В России ежегодно в результате неонатального скрининга на врожденный гипотиреоз (ВГ) диагностируется свыше 350 новых случаев заболевания: от 356 случаев в 2006 г. до 534 случаев в 2014 г. Отмечается стойкая тенденция к увеличению вновь выявленных случаев ВГ, связанная как с увеличением рождаемости и возрастающей долей охвата новорожденных скринингом, с одной стороны, так и с сохраняющейся проблемой дефицита йода разной степени выраженности на всей территории страны - с другой стороны [7, 9].

По данным анализа, проведенного ФГБУ «НМИЦ эндокринологии» Минздрава России за 1997-2015 гг,. в Российской Федерации в результате неонатального скрининга выявлено 6867 детей с ВГ (с 1997 г. в среднем в каждом субъекте РФ выявляется 4,76 случая ВГ в год); заболеваемость составила 1:3617 новорожденных. Наибольшая заболеваемость ВГ в России наблюдается в Уральском ФО и составляет 1:2379 (этот показатель в регионе регистрируется практически на протяжении всего периода наблюдения — от 1:1711 в 1999 г. до 1:3231 в 2015 г.) [10]. Обобщенные данные по охвату новорожденных и выявленным случаям заболевания в ФО представлены в табл. 1.

За последние годы ситуация с охватом новорожденных процедурой скрининга на ВГ в Крыму значительно улучшилась: по данным Минздрава России, в 2019 г. доля обследованных новорожденных составила 98,2 и 100\% в целом по республике и в городе федерального значения Севастополь соответственно; диагноз ВГ в 2019 г. был установлен одному ребенку [7].

Учитывая отсутствие в РФ закона о всеобщем йодировании соли, многие регионы до сих пор находятся в условиях умеренного или тяжелого йодного дефицита, и использование скрининга неонатального ТТГ в данных регионах возможно для оценки статуса йодного питания населения [9]. 
Таблица 1. Охват новорожденных скринингом и заболеваемость врожденным гипотиреозом по федеральному округу (совокупно за 1997-2015 гг., исключая 2005 г.)

\begin{tabular}{lccc}
\hline \multicolumn{1}{c}{ Фо } & $\begin{array}{c}\text { Доля охвата скринингом, } \\
\text { \% }\end{array}$ & $\begin{array}{c}\text { Количество выявленных } \\
\text { случаев }\end{array}$ & Заболеваемость \\
\hline Уральский & 93,3 & 991 & $1: 2379$ \\
Приволжский & 95,6 & 1533 & $1: 3376$ \\
Центральный & 99,9 & 1681 & $1: 3665$ \\
Сибирский & 93,9 & 962 & $1: 3875$ \\
Южный & 97,7 & 560 & $1: 4107$ \\
Северо-Западный & 96,0 & 520 & $1: 4271$ \\
Дальневосточный & 96,0 & 265 & $1: 4508$ \\
Северо-Кавказский & 81,7 & 350 & $1: 4752$ \\
Крымский (только 2015 г.) & 82,5 & 5 & $1: 4891$ \\
\hline
\end{tabular}

С целью понимания текущей ситуации по йодной обеспеченности населения Республики Крым по поручению Минздрава России ФГБУ «НМИЦ эндокринологии» Минздрава России в сентябре 2020 г. провел контрольноэпидемиологическое исследование. Исследований подобного рода в Крыму ранее не проводилось.

\section{ЦЕЛЬ ИССЛЕДОВАНИЯ}

Оценка текущей йодной обеспеченности населения Республики Крым.

\section{МАТЕРИАЛЫ И МЕТОДЫ}

\section{Место и время проведения исследования}

Республика Крым с марта 2014 г. является субъектом Российской Федерации, входящим в настоящее время в состав Южного федерального округа. Площадь территории региона составляет 0,15\% (26,1 тыс. кВ. Км) территории Российской Федерации с населением 2361760 постоянных жителей (по данным Росстата на 01.01.2020 г.).

Крымский полуостров отличается особым приморским положением, плодородными почвами, теплым климатом, а также наличием рекреационных ресурсов. Для Крыма характерно неравномерное распределение запасов пресных подземных вод. Так, более высокая (более 70 тыс. м³/сут) насыщенность геологических образований пресными водами установлена в Сакском, Джанкойском, Нижнегорском, Красногвардейском, Раздольненском, Бахчисарайском районах и на территории Севастопольского городского совета, тогда как в остальных районах запасы их значительно ниже. Йодобромные воды развиты в северной части Крыма на границе с Херсонской областью (Северо-Сивашское месторождение), в Сакском и Красногвардейском районах. По Северо-Сивашскому месторождению утверждены запасы промышленных йодных вод для производства йода, а по Новоселовскому - запасы термальных вод. Йодобромные воды этих месторождений в настоящее время используются в бальнеологических целях [11]. По данным Крымской республиканской ассоциации «Экология и мир», по результатам исследования в рамках проекта «Чистая вода для регионов Крыма», в воде ряда источников Крыма (по всей технологической цепочке обработки воды в Большой Ялте, г. Симферополе, г. Евпатории, г. Севастополе, г. Феодосии) определено низкое содержание фтора и йода. Анализы проб воды из 40 источников водохранилищ Республики Крым показали, что в преобладающем большинстве из них (n=37) низкие значения содержания йода, и только в 3 пробах концентрация йода находится «на нижней отметке физиологической нормы» (предельно допустимая концентрация (ПДК) 0,125 мг/л) [12].

Исследование проводилось с 7 по 11 сентября 2020 г. в следующих городах Республики Крым: Симферополе, Белогорске, Бахчисарае и Саки. Характеристики географического расположения районов исследования представлены в таблице 2.

Обследование детей осуществлялось в шести средних общеобразовательных школах (СОШ) региона: в г. Симферополе на базе МБОУ «СОШ №24», МБОУ «СОШ №7», МБОУ «СОШ №5», в г. Белогорске - МБОУ

Таблица 2. Особенности географического расположения районов исследования

\begin{tabular}{|c|c|c|c|c|}
\hline & $\begin{array}{c}\text { г. Саки } \\
\text { (Сакский район) }\end{array}$ & $\begin{array}{c}\text { г. Бахчисарай } \\
\text { (Бахчисарайский } \\
\text { район) }\end{array}$ & $\begin{array}{c}\text { г. Симферополь } \\
\text { (городской округ) }\end{array}$ & $\begin{array}{c}\text { г. Белогорск } \\
\text { (Белогорский } \\
\text { район) }\end{array}$ \\
\hline Расположение & Западное побережье & $\begin{array}{l}\text { Юго-западная } \\
\text { часть }\end{array}$ & Центральная часть & Центральная часть \\
\hline $\begin{array}{l}\text { Высота над уровнем } \\
\text { моря, м }\end{array}$ & От 7 до 33 м & $\begin{array}{l}\text { От } 97 \text { до } 300 \text { м } \\
\text { для г. Бахчисарая }\end{array}$ & $\begin{array}{l}\text { От } 20 \text { до } 445 \text { м, } 300 \text { м } \\
\text { для г. Симферополя }\end{array}$ & $\begin{array}{l}\text { От } 60 \text { до } 564 \text { м, } 180 \text { м } \\
\text { для г. Белогорска }\end{array}$ \\
\hline $\begin{array}{l}\text { Тип рельефа } \\
\text { местности }\end{array}$ & Равнинный & Холмистый & Холмистый & $\begin{array}{l}\text { Холмистый } \\
\text { (предгорье) }\end{array}$ \\
\hline
\end{tabular}


«Школа-лицей № 2», в г. Бахчисарае - МБОУ «СОШ №7», в г. Саки - МБОУ «Сакская средняя школа №1 им. Героя Советского Союза В.К. Гайнутдинова». Выбор населенных пунктов и учреждений выполнялся с учетом количества обучающихся и возможности обследовать не менее 30 школьников в возрасте 8-10 лет. Выборка была сформирована методом систематического выбора с учетом обучения в школах не только детей, проживающих в данном городе, но и приезжающих из других населенных пунктов региона.

Дизайн исследования: одномоментное популяционное исследование.

\section{МЕТОДЫ}

Обследовано 356 детей допубертатного возраста (8-10 лет), всем были выполнены: сбор анамнеза и антропометрических показателей (рост, вес), осмотр врача-эндокринолога с пальпацией ЩЖ, УЗИ ЩЖ, получение разовых порций мочи и образцов пищевой поваренной соли (5-10 г), которая ежедневно используется в питании в их семьях.

Измерения роста и веса детей стандартной методикой проводились во время осмотра специалистом.

УЗИ ЩЖ выполнялось в соответствии с рекомендациями ВОЗ в положении лежа с использованием портативного ультразвукового аппарата LOGIQе (China) с мультичастотным линейным датчиком 10-15 МГц. Объем ЩЖ рассчитывался по формуле:

Vщж $=[($ Шпр. $x$ Дпр. $x$ Тпр. $)+$ (Шл. $x$ Дл. $x$ Тл.) $]$ x 0,479, где Ущж - объем щитовидной железы; Шпр. - ширина правой доли щитовидной железы; Шл. - ширина левой доли щитовидной железы; Дпр. - длина правой доли щитовидной железы; Дл. - длина левой доли щитовидной железы; Тпр. - толщина правой доли щитовидной железы; Тл. - толщина левой доли щитовидной железы.

У детей соответствие объема ЩЖ нормативным показателям, разработанным Zimmermann М. и соавт., оценивалось с учетом площади поверхности тела и пола [13].

Bсе образцы мочи $(n=356)$ в одноразовых микропробирках типа Эппендорф сразу же после получения подвергались заморозке при температуре $-20-25^{\circ} \mathrm{C}$ для дальнейшей транспортировки в лабораторию. Концентрация йода в моче определялась церий-арсенитным методом на базе клинико-диагностической лаборатории ФГБУ «НМИЦ эндокринологии» Минздрава России. Данная методика обеспечивает выполнение измерений концентрации общего йода в моче в диапазоне концентраций 20-300 мкг/л (0,16-2,37 мкмоль/л). Общий йод представляет собой сумму связанного йода и свободного йодида [14].

Качественное исследование на наличие йодата калия в образцах пищевой поваренной соли $(n=203)$, которая используется в семьях школьников, осуществлялось на месте экспресс-методом. Принцип метода заключается в изменении окраски раствора крахмала при выделении свободного йода из соли после обработки ее тест-раствором. Степень изменения окраски оценивается визуально.

От всех родителей/опекунов детей получены информированные согласия на проведение обследования и обработку персональных данных.

\section{Статистический анализ}

Данные представлены в виде абсолютных значений и процентов от общего количества. Для описательного статистического анализа концентрации йода в моче были использованы значения медианы и частотного распределения.

\section{Этическая экспертиза}

Протокол исследования одобрен на заседании этического комитета. Разрешение локального этического комитета ФГБУ «НМИЦ эндокринологии» Минздрава России получено 25 марта 2020 г., №5.

\section{РЕЗУЛЬТАТЫ}

По результатам проведенного в Крыму исследования мКЙМ составляет 97 мкг/л и варьирует от 78 до 98 мкг/л в обследованных районах (табл. 3). Доля проб мочи со сниженной концентрацией йода составила 51,2\%, при этом уровень йода 50-49 мкг/л определен в 41,6\% проб, а уровень <50 мкг/л - в 9,6\%, что не позволяет считать потребление йода достаточным и соответствует йодному дефициту легкой степени. Частотное распределение концентрации йода в моче в обследованной популяции представлено на рисунке 1.

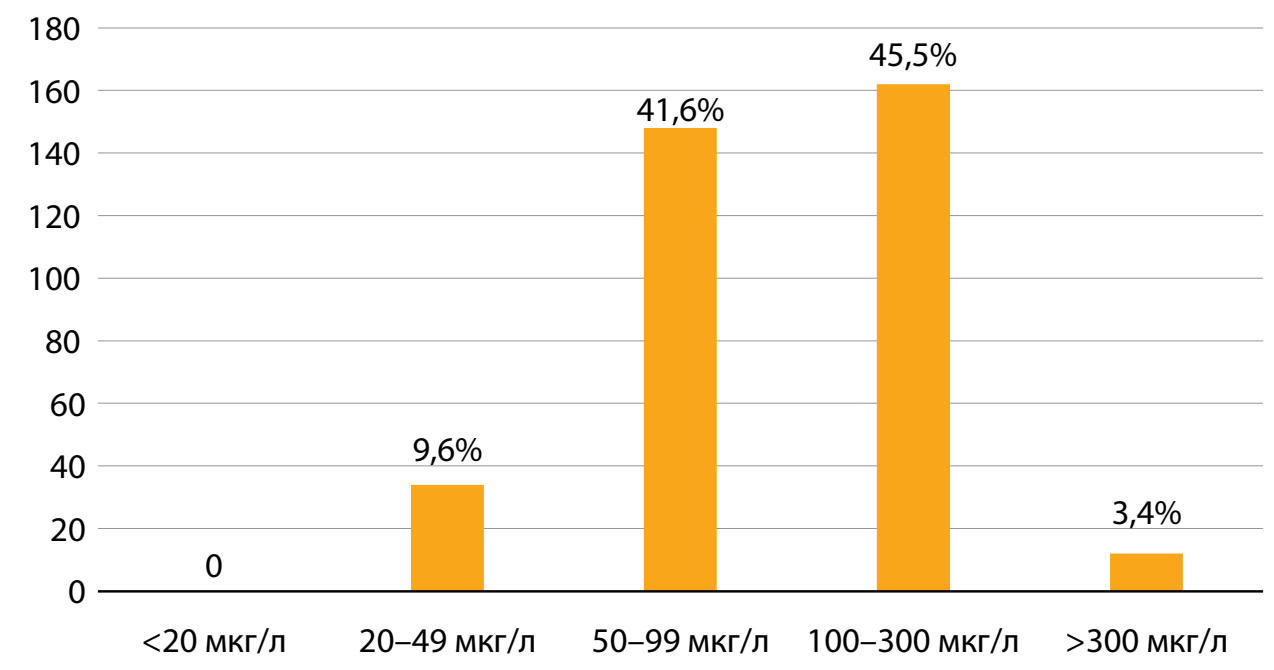

Рисунок 1. Частотное распределение концентрации йода в моче у обследованных школьников. 

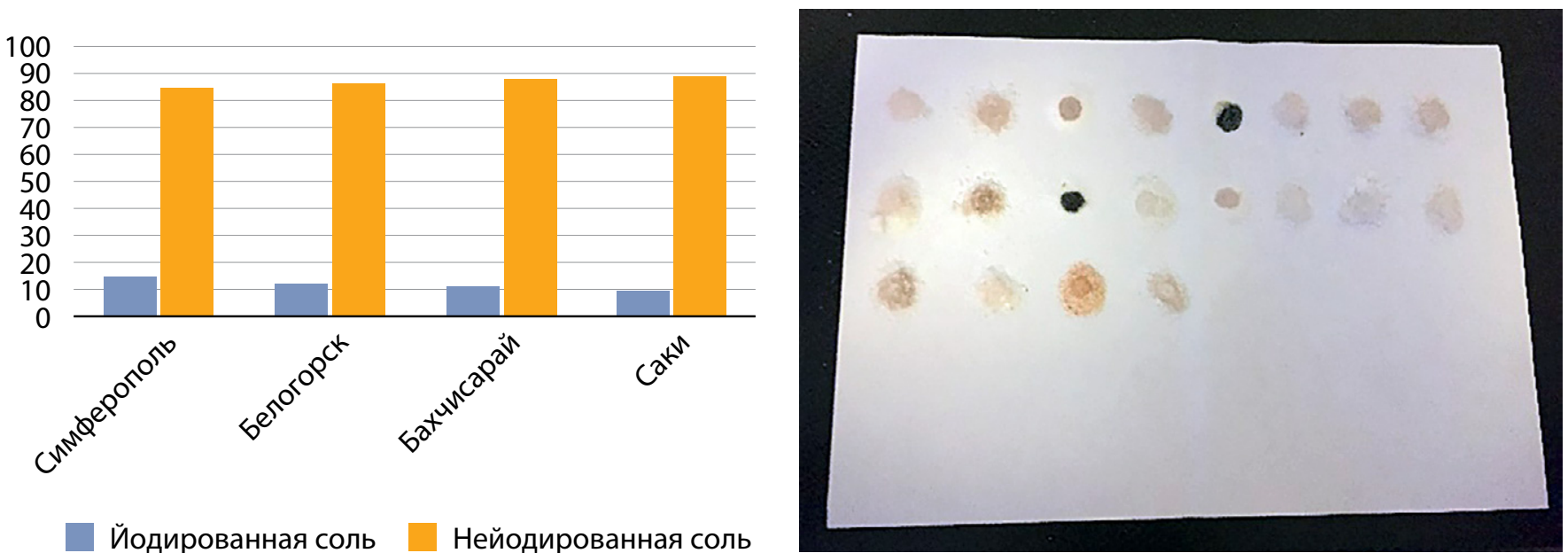

Йодированная соль Нейодированная соль

Рисунок 2. Результаты определения наличия йода в пищевой соли из домохозяйств и вид йодированной соли при проведении экспресс-теста (синее окрашивание образцов соли подтверждает наличие йодата калия).

Во всех районах, в которых проводилось исследование, только 12,3\% исследуемых образцов соли оказались йодированными (диапазон от 10 до 15\%), что свидетельствует о крайне низком уровне потребления йодированной соли населением (рис. 2).

По данным УзИ ЩЖ у 9,5\% обследованных детей был выявлен диффузный 30б, частота зоба в исследуемой выборке варьировала от 1,7 до 16,3\%. Результаты проведенных исследований в населенных пунктах Республики Крым представлены в таблице 3.

\section{ОБСУЖДЕНИЕ}

До 1950 г. эндемический зоб на Украине имел достаточно широкое распространение, в первую очередь в западных областях (предгорья Карпатских гор). В период с 1950 по 1980 гг. в стране стали проводиться активные профилактические мероприятия, в результате которых эта проблема перестала быть актуальной [15].

Распад Советского Союза в 1991 г. стимулировал попытки создания законодательной базы в ряде

Таблица 3. Результаты исследований в населенных пунктах Республики Крым

\begin{tabular}{|c|c|c|c|}
\hline Населенный пункт & $\begin{array}{l}\text { Распространенность } \\
\text { диффузного зоба, \% }\end{array}$ & мКЙМ, мкг/л & $\begin{array}{c}\text { Доля йодированной } \\
\text { соли, \% }\end{array}$ \\
\hline г. Симферополь & 1,7 & 98 & 15 \\
\hline г. Белогорск & 6,6 & 95 & 12,7 \\
\hline г. Бахчисарай & 10,4 & 84 & 11,5 \\
\hline г. Саки & 16,3 & 78 & 10 \\
\hline
\end{tabular}
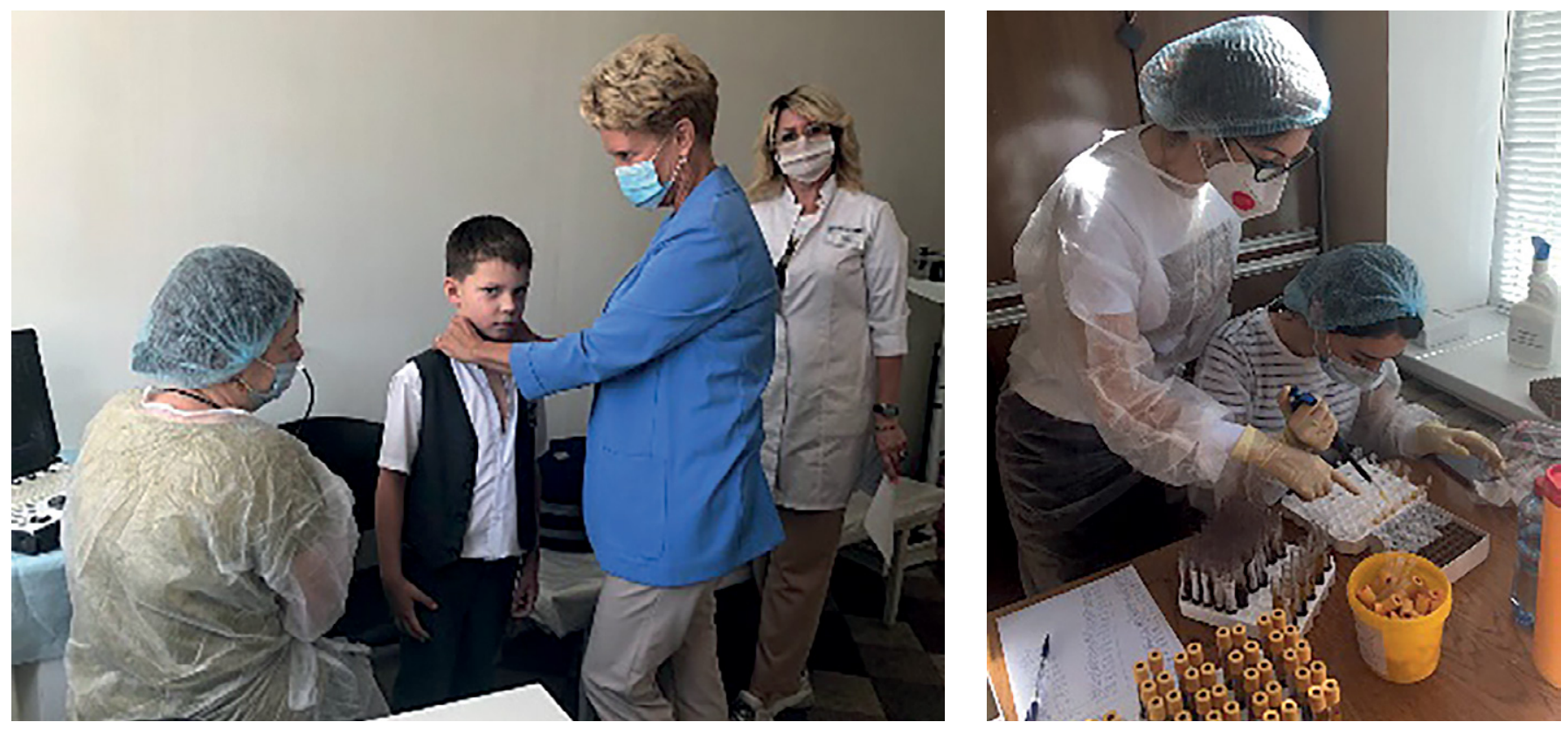

Рисунок 3. Сотрудники ФГБУ «НМИЦ эндокринологии» проводят обследование детей в Республике Крым. 
постсоветских стран, в том числе и на Украине. В 1997 г. правительство Украины приняло постановление «О мерах по профилактике йододефицитных заболеваний», однако существовавшие нормативные акты предусматривали добровольную модель, отсутствовали четкие механизмы обеспечения йодированной солью населения, что привело к низкому уровню производства и поставок йодированной соли [16]. Согласно данным общенациональных исследований, проведенных в 2002 и 2005 гг., только 18-20\% домохозяйств на Украине использовали в питании качественную йодированную соль [15]. В 2005 г. Министерством здравоохранения АР Крым предпринимались попытки осуществления профилактических мероприятий, направленных на решение проблемы ЙД3. Однако в действительности такая профилактика заключалась в применении биологически активных добавок на основе йодированных белков коровьего молока, что в итоге, как и следовало ожидать, не дало никаких результатов [17]. Коммуникационная и информационная деятельность ЮНИСЕФ и Глобальной сети по йоду (GAIN) в Украине привела к тому, что в 2012 г. две зарегистрированные средние и крупные пекарни, расположенные во Львове и Киеве, изменили свои производственные процессы на использование йодированной соли в выпечке хлеба [16]. Объем производства хлеба обеспечивал 19000 человек (около 0,04\% населения), исходя из среднего потребления - 240 г хлеба на душу населения в день, при этом содержание йодированной соли в конечном продукте составило 70\% [16].

Крымский полуостров оказался территорией, которую проводимые профилактические мероприятия практически не затронули, и по настоящее время в республике нет действующих программ профилактики ЙДЗ.

Считается, что статус йодной обеспеченности детей школьного возраста является индикативным для всей популяции территории (исключение составляют беременные и кормящие женщины). Однако после принятия в 2019 г. нового СанПиН 2.4.5.2409-08, предусматривающего обязательное использование йодированной соли в школьном питании с начала 2020 г., показатели экскреции йода с мочой у детей школьного возраста могут неточно отражать обеспеченность йода во всей популяции. В этой связи умеренное снижение мКЙМ (97 мкг/л) не позволяет считать потребление йода достаточным для всего населения, особенно на фоне крайне низкого уровня использования пищевой йодированной соли в домохозяйствах - только $12,3 \%$. Четко прослеживается и показательна прямая зависимость возрастания мКЙМ при повышении доли употребления йодированной соли и обратная корреляция этих показателей с частотой выявления зобных изменений ЩЖ по данным УЗИ. Напряженная ситуация с потреблением йода имеет место в Сакском районе, где и были установлены минимальный показатель мКЙМ - 78 мкг/л и максимальная частота распространенности зоба у детей - 16,3\% (при этом интересен факт, что данный район относится к равнинной приморской территории), а наиболее благоприятная эпидемиологическая ситуация - в центральной части Республики Крым и г. Симферополе, для которого мКЙМ составляет 98 мкг/л, доля зоба у детей - 1,7\%.

Несоответствие «субнормального» значения мКЙМ и низкого процента йодированной соли во всех иссле- дованных районах послужит поводом для проведения новых поисково-аналитических и эпидемиологических исследований, включая определение экскреции йода у беременных и женщин репродуктивного возраста и изучение структуры питания городского и сельского населения в этом регионе страны.

Однако очевидно, что для устойчивой ликвидации дефицита йода у всего населения недостаточно полагаться на пропаганду употребления йодированной соли на уровне домохозяйств. Результаты уже упомянутого выше двойного слепого рандомизированного контролируемого исследования, проведенного в г. Донецке (Украина), показали, что замена в домохозяйствах обычной соли на йодированную уже через 2 нед нормализовала йодный статус у детей (мКЙМ увеличилась со 102 до 169 мкг/л), но не у беременных женщин [15]. Для устойчивой нормализации йодного статуса населения важно также использование йодированной соли в пищевой промышленности, особенно в производстве хлебобулочных изделий.

Согласно данным Глобальной сети по йоду, Украина, наряду с Россией, по-прежнему входит в число стран, имеющих дефицит йода (список которых значительно сократился с 1990 по 2017 гг. - со 113 до 20 соответственно), в которых отсутствуют государственные программы по йодированию соли. В отличие от Беларуси, где с 2001 г. действует государственная программа по профилактике ЙД3, по результатам исследований 2018 г. мКЙМ в популяции школьников 9-12 лет составляет 191 мкг/л, и обеспеченность населения йодом на этой территории в настоящее время можно считать оптимальной $[4,18]$.

\section{выводы}

Полученные результаты исследования свидетельствуют о недостаточной обеспеченности населения Республики Крым йодом. мКЙМ составляет 97 мкг/л, что отражает неадекватное йодное обеспечение и соответствует йодному дефициту легкой степени.

\section{Клиническая значимость результатов}

Клиническая значимость полученных результатов состоит в комплексной оценке состояния йодной обеспеченности и зобной эндемии и необходимости принятия программ профилактики ЙДЗ на территории Республики Крым на законодательном уровне.

\section{Ограничения исследования}

Проведено пилотное исследование с включением ряда городов и столицы региона, для более точной оценки эпидемиологической ситуации требуется включение большего количества районов при согласовании с Министерством здравоохранения Республики Крым.

\section{Направления дальнейших исследований}

В продолжение работы планируются сопоставление полученных данных с результатами неонатального скрининга на ВГ, а также исследования йодной обеспеченности беременных и кормящих женщин на фоне массовой йодной профилактики, уточнение и научное обоснование необходимости дополнительной йодной дотации в виде лекарственных препаратов йода данным группам населения. 


\section{ЗАКЛЮЧЕНИЕ}

Мониторинг ЙДЗ и йодной обеспеченности в Республике Крым показал сохранение йодного дефицита в регионе при отсутствии четких программ йодной профилактики, прежде всего массовой профилактики йодированной солью, даже с учетом географического положения. Таким образом, полученные результаты контрольно-эпидемиологического исследования еще раз подтверждают необходимость принятия и реализации закона о профилактике ЙДЗ в России посредством употребления йодированной соли [19].

\section{ДОПОЛНИТЕЛЬНАЯ ИНФОРМАЦИЯ}

Источники финансирования. Исследование выполнено при финансовой поддержке государственных заданий: «Научная оценка необходимости принятия дополнительных нормативных правовых и иных мер по ликвидации йодного дефицита в пилотных регионах с тяжелым йодным дефицитом», рег. № 860000Ф.99.1.АЕ32АА00001 (фрагмент, посвященный контрольно-эпидемиологическим исследованиям в регионе); «Эпидемиологические и молекулярно-клеточные характеристики опухолевых, аутоиммунных и йододефицитных ти реопатий как основа профилактики осложнений и персонализации лечения», рег. № АAАA-А20-120011790180-4 (аналитический обзор мероприятий, предшествующих контрольно-эпидемиологическим исследованиям в регионе).
Конфликт интересов. Авторы декларируют отсутствие явных и потенциальных конфликтов интересов, связанных с публикацией настоящей статьи.

Участие авторов. Трошина Е.А., Абдулхабирова Ф.М., Маколина Н.П., Никанкина Л.В., Малышева Н.М., Репинская И.Н., Дивинская В.А. - концепция и дизайн исследования; Сенюшкина Е.С. - сбор и обработка материалов, анализ полученных данных, написание текста. Все авторы одобрили финальную версию статьи перед публикацией, выразили согласие нести ответственность за все аспекты работы, подразумевающую надлежащее изучение и решение вопросов, связанных с точностью или добросовестностью любой части работы.

Благодарности. За содействие реализации исследования авторы выражают признательность аппарату Правительства Республики Крым в лице заместителя Председателя Совета министров Республики Крым - министру труда и социальной защиты Республики Крым Романовской Елене Васильевне, министру здравоохранения Республики Крым Чемоданову Игорю Геннадьевичу (до 09.2020 г.), министру образования, науки и молодежи Республики Крым Лаврик Валентине Васильевне; за всестороннее участие в подготовке, координации и проведении исследования - главному внештатному специалисту эндокринологу Министерства здравоохранения Республики Крым Репинской Ирине Николаевне и главному внештатному детскому специалисту эндокринологу Министерства здравоохранения Республики Крым Дивинской Валентине Александровне, руководству ГБУз РК «РКБ имени Н.А. Семашко» в лице главного врача Остапенко Александра Ивановича, руководителям медицинских организаций и директорам общеобразовательных школ в районах проведения исследования.

\section{СПИСОК ЛИТЕРАТУРЫ | REFERENCES}

1. Трошина Е.А., Платонова Н.М., Абдулхабирова Ф.М., Герасимов Г.А Йододефицитные заболевания в Российской Федерации: время принятия решений / Под ред. И.И. Дедова, Г.А. Мельниченко. М.: OAO «Конти-Принт»; 2012. 232 с. [Troshina EA, Platonova NM, Abdulkhabirova FM, Gerasimov GA. Iododefitsitnye zabolevaniya $\checkmark$ Rossiiskoi Federatsii: vremya prinyatiya reshenii. Ed. by Dedov II, Mel'nichenko G. Moscow: OAO «Konti-Print»; 2012. 232 p. (In Russ.)].

2. Мельниченко Г.А., Трошина Е.А., Платонова Н.М., и др.

Осведомленность населения России о йододефицитных заболеваниях и способах их профилактики // Клиническая и экспериментальная тиреоидология. — 2016. - Т. 12. №3. - C. 25-30. [Melnichenko GA, Troshina EA, Platonova NM, et al. The awareness of Russians about iodine deficiency diseases and methods of it's prevention. Clinical and experimental thyroidology. 2017;12(3):25-30. (In Russ.)]. doi: https://doi.org/10.14341/ket2016325-30

3. Герасимов Г.А., Фадеев В.В., Свириденко Н.Ю., и др. Йоддефицитные заболевания в России. Простое решение сложной проблемы. М.: Адаманть; 2002. 168 с. [Gerasimov GA, Fadeev W, Sviridenko NYu, et al. loddefitsitnye zabolevaniya $v$ Rossii. Prostoe reshenie slozhnoi problemy. Moscow: Adamant'; 2002. 168 p. (In Russ.)].

4. Алферова В.И., Мустафина С.В., Рымар О.Д. Йодная обеспеченность в России и мире: что мы имеем на 2019 год? // Клиническая и экспериментальная тиреоидология. — 2019. — Т. 15. — №2. - 73-82. [Alferova VI, Mustafina SV, Rymar OD. lodine status of the population in Russia and the world: what do we have for 2019? Clin Exp Thyroidol. 2019;15(2):73-82. (In Russ.)]. doi: https://doi.org/10.14341/ket10353

5. Алферова В.И., Мустафина С.В., Рымар О.Д. Рекомендации по мониторингу программ йодирования соли и оценке статуса йодной обеспеченности населения (русскоязычная версия) // Клиническая и экспериментальная тиреоидология. - 2018. T. 14. — №2. — C. 100-112. [Alferova VI, Mustafina SV, Rymar OD Guidance on the monitoring of salt iodization programmes and determination of population iodine status: Russian language version Clinical and experimental thyroidology. 2018;14(2):100-112. (In Russ.)]. doi: https://doi.org/10.14341/ket9734

6. Здравоохранение в России. 2019: Стат. сб./Росстат. - М.: 3-46; 2019. 170 с. Доступно по: https://rosstat.gov.ru/storage/mediabank/
Zdravoohran-2019.pdf [Zdravookhranenie v Rossii. 2019: Stat. sb.] Rosstat. Moscow: Z-46; 2019. 170 p. Available from: https://rosstat. gov.ru/storage/mediabank/Zdravoohran-2019.pdf (In Russ.)].

7. Государственный доклад Межрегионального управления Роспотребнадзора по Республике Крым и городу федерального значения Севастополю «О состоянии санитарноэпидемиологического благополучия населения в Республике Крым и городе федерального значения Севастополе в 2019 году». Доступно по: http://82.rospotrebnadzor.ru/documents/Gosdoklad [Gosudarstvennyi doklad Mezhregional'nogo Upravleniya Rospotrebnadzora po Respublike Krym i gorodu federal'nogo znacheniya Sevastopolyu «O sostoyanii sanitarno-epidemiologicheskogo blagopoluchiya naseleniya $\vee$ Respublike Krym i gorode federal'nogo znacheniya Sevastopole v 2019 godu». Available from: http://82.rospotrebnadzor.ru/documents/Gosdoklad (In Russ.)].

8. Энзель Д.А., Славников А.А., Иванов С.В. Распространенность заболеваний щитовидной железы в Судакском районе Республики Крым за период 2016-2018 гг. // ВосточноЕвропейский научный журнал. Медицинские науки. - 2020. T. 53. - №2. - C. 27-29. [Enzel'DA, Slavnikov AA, Ivanov SV. Raspostranennost'zabolevanii shchitovidnoi zhelezy v Sudakskom raione Respubliki Krym za period 2016-2018 gg. Vostochno Evropeiskii Nauchnyi Zhurnal. Meditsinskie nauki. 2020;53(2):27-29. (In Russ.)].

9. Трошина Е.А., Рыбакова А.А., Куцев С.И., и др. Информативность эпидемиологических показателей в оценке йодной обеспеченности населения (на примере регионов РФ) // Архивъ внутренней медичины. - 2019. - T. 9. - №5. - C. 367-372. [Troshina EA Rybakova AA, Kutsev SI, et al. Epidemiological indicator value in the iodine availability assessment — evidence from the region of the Russian Federation. Russ Arch Intern Med. 2019;9(5):367-372. (In Russ.)]. doi: https://doi.org/10.20514/2226-6704-2019-9-5-367-372

10. Дедов И.И., Безлепкина О.Б., Вадина Т.А., и др. Скрининг на врожденный гипотиреоз в Российской Федерации // Проблемы эндокринологии. - 2018. - Т. 64. — №1. — С. 14-20. [Dedov II, Bezlepkina OB, Vadina TA, et al. Screening for congenital hypothyroidism in the Russian Federation. Problems of Endocrinology. 2018;64(1):14-20. (In Russ.)] doi: https://doi.org/10.14341/probl8752 
11. Доклад о состоянии и охране окружающей среды на территории Республики Крым в 2015 г. Министерство экологии и природных ресурсов Республики Крым. Доступно по: https://meco.rk.gov. ru/rus/file/Doklad_o_sostojanii_i_ohrane_okruzhajushhej_sredy_ Respubliki_Krym_v_2015.pdf [Doklad o sostoyanii i okhrane okruzhayushchei sredy na territorii Respubliki Krym v 2015 godu. Ministerstvo ekologii i prirodnykh resursov Respubliki Krym. Available from: https://meco.rk.gov.ru/rus/file/Doklad_o_sostojanii_i_ohrane_ okruzhajushhej_sredy_Respubliki_Krym_v_2015.pdf (In Russ.)].

12. Иванов С.В., Гук М.Г., и др. Взаимосвязь химического состава почвы и поверхностных вод Республики Крым и их влияние на развитие эндемических заболеваний // Центральный научный вестник. 2018. — T. 3. — №10. — C. 15-19. [Ivanov SV, Guk MG. Relationship of chemical composition of soil and surface waters of the Republic of Crimea and their influence on development of endemic diseases. Tsentral'nyi nauchnyi vestnik. 2018;3(10):15-19. (In Russ.)].

13. Zimmermann MB, Hess SY, Molinari $L$, et al. New reference values for thyroid volume by ultrasound in iodine-sufficient schoolchildren: a World Health Organization/Nutrition for Health and Development lodine Deficiency Study Group Report. Am J Clin Nutr. 2004;79(2):231-237. doi: https://doi.org/10.1093/ajcn/79.2.231

14. Дедов И.И., Арбузова М.И., Ильин А.В. Йодная лаборатория Приниипы организачии работы. Пособие для врачейлаборантов. - M.; 2005. 48 с. [Dedov II, Arbuzova MI, Il'in AV. lodnaya laboratoriya. Printsipy organizatsii raboty. (posobie dlya vracheilaborantov). Moscow; 2005. 48 p. (In Russ.)].

15. Фирсова Н.А., ван дер Хаар Ф., Демина Т.Н., и др. Использование йодированной соли в домашнем хозяйстве улучшает

обеспечение питания йодом у беременных и детей школьного возраста: двойное слепое рандомизированное контролируемое исследование в Донецке // Клиническая и экспериментальная тиреоидология. — 2011. - Т. 7. - №2. - C. 33-42. [Firsova NA, van der Khaar F, Demina TN, et al. Use of iodized salt in the households improves the iodine status of pregnant women and school-age children in Donetsk: a double-blind randomized controlled trial. Clinical and experimental thyroidology. 2011;7(2):33-42. (In Russ.)]. doi: https://doi.org/10.14341/ket20117233-42

16. Knowles J, van der Haar F, Shehata M, et al. lodine Intake through Processed Food: Case Studies from Egypt, Indonesia, the Philippines, the Russian Federation and Ukraine, 2010-2015. Nutrients. 2017;9(8):797. doi: https://doi.org/10.3390/nu9080797

17. Донич С.Г., Игнатонис Й.П., Дударь Л.В., и др. Метод йодорганической коррекции питания (профилактика и лечение заболеваний, связанных с недостатком йода). Методические рекомендации. Симферополь; 2005. [Donich SG, Ignatonis IP, Dudar' LV, et al. Metod iodorganicheskoi korrektsii pitaniya (profilaktika i lechenie zabolevanii, svyazannykh s nedostatkom ioda). Metodicheskie rekomendatsii. Simferopol'; 2005. (In Russ.)].

18. Мохорт Т.В., Петренко С.В., Леушев Б.Ю., и др. Оценка йодного обеспечения детей школьного возраста и беременных женщин в Республике Беларусь в 2017-2018 гг. // Клиническая и экспериментальная тиреоидология. - 2018. - Т. 14. - №3. C. 149-155. [Mokhort TV, Petrenko SV, Leushev BY, et al. Assessment of iodine status among school age children and pregnant women of Belarus in 2017-2018. Clinical and experimental thyroidology. 2018;14(3):149-155. (In Russ.)]. doi: https://doi.org/10.14341/ket9732

19. Проект Федерального закона «О профилактике заболеваний, связанных с дефицитом йода». Доступно по: https://rosstat. gov.ru/ D1\%82\%D0\%B0\%D0\%BB\%D1\%8C\%D0\%BD\%D1\% 8B\%D0\%B9+\%D1\%81\%D0\%BA\%D1\%80\%D0\%B8\%D0\%BD\%D0\%B8\%D0\%BD\%D0\%B3+\%D1\%82\%D1\%82\%D0\%B3 [Proekt Federal'nogo zakona «O profilaktike zabolevanii, svyazannykh s defitsitom ioda». Available from: https://rosstat.gov.ru/ D1\%82\%D0\%B0\%D0\%BB\%D1\%8C\%D0\%BD\%D1\%8B\%D0\%B9+ \%D1\%81\%D0\%BA\%D1\%80\%D0\%B8\%D0\%BD\%D0\%B8\%D0\%BD\%D0\%B3+\%D1\%82\%D1\%82\%D0\%B3 (In Russ.)].

\section{ИНФОРМАЦИЯ ОБ АВТОРАХ [AUTHORS INFO]}

*Сенюшкина Евгения Семеновна, н.с. [Evgeniya S. Senyushkina, MD]; адрес: Россия, 117036, Mосква, ул. Дм. Ульянова, д. 11 [address: 11 Dm. Ulyanova street, 117036 Moscow, Russia];

ORCID: https://orcid.org/0000-0001-7960-8315; eLibrary SPIN: 4250-5123; e-mail: EvgeniyaSenyushkina@yandex.ru

Трошина Екатерина Анатольевна, д.м.н., член-корр. РАН, професcop [Ekaterina A. Troshina, MD, PhD, Professor]; ORCID: https://orcid.org/0000-0002-8520-8702; eLibrary SPIN: 8821-8990; e-mail: troshina@inbox.ru

Абдулхабирова Фатима Магомедовна, к.м.н. [Fatima M. Abdulkhabirova, MD, PhD];

ORCID: https://orcid.org/0000-0001-8580-2421; eLibrary SPIN: 2462-1115; e-mail: a-fatima@yandex.ru

Маколина Наталья Павловна, н.C. [Natalya P. Makolina, MD]; ORCID: https://orcid.org/0000-0003-3805-7574;

eLibrary SPIN: 7210-9512; e-mail: makolina.natalia@endocrincentr.ru

Никанкина Лариса Вячеславовна, к.м.н. [Larisa V. Nikankina, PhD]; ORCID: https://orcid.org//0000-0001-8303-3825; eLibrary SPIN: 2794-0008; e-mail: nikankina.larisa@endocrincentr.ru

Малышева Наталья Михайловна, к.б.н. [Natalia M. Malysheva, PhD]; ORCID: https://orcid.org/0000-0001-7321-9052; eLibrary SPIN: 5793-2550; e-mail: Malysheva.Natalya@endocrincentr.ru

Репинская Ирина Николаевна, врач-эндокринолог [Irina N. Repinskaya, MD]; ORCID: 0000-0001-6200-1699;

eLibrary SPIN: 8171-9212; e-mail: repinskaya.irina@mail.ru

Дивинская Валентина Александровна, к.м.н., доцент, професcop [Valentyna A. Divinskaya, Ph.D., Professor]; ORCID: 000-0001-5952-937X; eLibrary SPIN: 2063-7214; e-mail: artishenko-valen@mail.ru

\section{ЦИТИРОВАТЬ}

Трошина Е.А., Сенюшкина Е.С., Маколина Н.П., Абдулхабирова Ф.М., Никанкина Л.В., Малышева Н.М., Репинская И.Н., Дивинская В.А. Йододефицитные заболевания: текущее состояние проблемы в Республике Крым // Клиническая и экспериментальная тиреоидология. — 2020. — Т.16. — №4. - С. 19-27.

doi: https://doi.org/10.14341/ket12700

Рукопись получена: 13.02.2021. Одобрена к публикации: 24.03.2021

\section{TO CITE THIS ARTICLE}

Troshina EA, Senyushkina ES, Makolina NP, Abdulkhabirova FM, Nikankina LV, Malysheva NM, Repinskaya IN, Divinskaya VA. lodine Deficiency Disorders: Current State of the Problem in the Republic of Crimea. Clinical and experimental thyroidology. 2020;16(4):19-27. doi: https://doi.org/10.14341/ket12700

Received: 13.02.2021. Accepted: 24.03.2021 